(c) 2005 International Press

Adv. Theor. Math. Phys. 9 (2005) 703-728

\title{
Classical integrability and
}

\section{super Yangian of superstring on $\mathrm{AdS}_{5} \times \mathrm{S}^{5}$}

\author{
Machiko Hatsuda ${ }^{1,2}$ and Kentaroh Yoshida ${ }^{1}$
}

${ }^{1}$ Theory Division, High Energy Accelerator Research Organization (KEK), Tsukuba, Ibaraki 305-0801, Japan

mhatsuda@post.kek.jp, kyoshida@post.kek.jp

${ }^{2}$ Urawa University, Saitama 336-0974, Japan

\begin{abstract}
We discuss a classical integrability in the type IIB string theory on the $\mathrm{AdS}_{5} \times \mathrm{S}^{5}$ background. By using the Roiban-Siegel formulation of the superstring on the $\mathrm{AdS}_{5} \times \mathrm{S}^{5}$, we carefully treat the Wess-Zumino term and the constraint conditions intrinsic to the supersymmetric case and construct explicitly non-local charges for a hidden infinite-dimensional symmetry. The existence of the symmetry is shown by Bena-PolchinskiRoiban. Then the super Yangian algebra is calculated. We also show the Serre relation ensuring the structure of the Hopf algebra. In addition, the classical integrability is discussed by constructing the Lax pair and the transfer matrix.
\end{abstract}

e-print archieve: http://lanl.arXiv.org/abs/hep-th/0407044 


\section{Introduction}

One of the most important subjects in string theory is to test the AdS/CFT correspondence $[28,42,51]$ beyond supergravity (i.e., BPS sectors). In the analysis at almost BPS region, a great development was made by BerensteinMaldacena-Nastase (BMN) [10]. They presented the AdS/CFT correspondence at string theoretical level by using the Penrose limit and the exact solvability of the pp-wave string theory. The pp-wave string states and their energies correspond to the BMN operators and the full conformal dimensions (including the anomalous dimensions) in the $\mathcal{N}=4$ super Yang-Mills (SYM) side, respectively. After the BMN's work, an approach beyond the BMN limit was proposed via the semi classical quantization of string theory around a classical solution (sigma model approach) $[27,29]$ instead of the BPS null geodesics used in [10]. The correspondence of semiclassical string states and composite operators in $\mathcal{N}=4 \mathrm{SYM}$ is precisely formulated in [8], and it implies the AdS/CFT correspondence at non-BPS region.

In the SYM theory side, Minahan and Zarembo [44] found the integrable structure by studying the composite operators. They discussed the $\mathrm{SO}(6)$ sector in the $\mathcal{N}=4 \mathrm{SYM}$ and studied the operator mixing of local composite operators at one-loop planer level by using the standard perturbation theory with respect to the 't Hooft coupling $\lambda$ in the large $N$ limit. Then they found that one-loop planer anomalous dimension matrix can be expressed as a Hamiltonian of the $\mathrm{SO}(6)$ quantum Heisenberg spin chain. This spin chain can be solved by using the algebraic Bethe ansatz. The eigen-values and eigen-states give the anomalous dimensions and the corresponding operators. Further developments have been done, for example, these operators include chiral operators, Konishi operators, and the complete one-loop dilatation operators found by Beisert [6].

On the other hand, the integrable structure in the string theory side, which is related to non-local charges in two-dimensional sigma models, was discussed [40]. In 1978, Lüscher and Pohlmeyer [37] found an infinite set of conserved non-local charges in two-dimensional classical non-linear sigma models $\left(\mathrm{NLSM}_{2}\right)$. The existence of classical non-local charges leads to quantum non-local charges which give constraints on the S-matrix and factorize it. Hence, non-local charges are intimately connected to the classical and quantum integrabilities of the theories. These charges are related to the Kac-Moody algebra [21] and generate the Drinfel'd Yangian algebra [23] in the work [12] (For review of Yangian symmetry, see [13]). That is, the non-local charges give a quantum field realization of the Yangian. 
The existence of classical integrability and non-local charges in the type IIB string theory on the $\mathrm{AdS}_{5} \times \mathrm{S}^{5}$ was suggested in the early work [40] by regarding the $\mathrm{S}^{5}$ part as an NLSM. The bosonic coset, $\mathrm{SO}(2,4) / \mathrm{SO}(4,1) \times$ $\mathrm{SO}(6) / \mathrm{SO}(5)=\mathrm{AdS}_{5} \times \mathrm{S}^{5}$, is a symmetric space and so the $\mathrm{AdS}_{5} \times \mathrm{S}^{5}$ valued NLSM has non-local charges. Contrasting to that for a symmetric coset space the flat current and the non-local charges are studied well, for example, in [1], and for a non-symmetric coset space, general arguments have not been established yet so far. A coset space for a superstring system is not a symmetric space which reflects the superalgebra, $\{Q, Q\} \sim P$. For such a non-symmetric coset space $\mathrm{PSU}(2,2 \mid 4) /(\mathrm{SO}(1,4) \times \mathrm{SO}(5))$, BenaPolchinski-Roiban [9] showed the existence of non-local charges by constructing the flat current based on the Maurer-Cartan equation in the simpler notation of [48] and the field equations obtained in [43]. The GreenSchwarz (GS) superstring on the $\mathrm{AdS}_{5} \times \mathrm{S}^{5}$ contains the Wess-Zumino (WZ) term and the $\kappa$-symmetry. Unlike the sigma models with the supergroup target spaces [14], the GS action does not contain fermionic currents in the kinetic term but only in the WZ term, so the superstring system has the fermionic constraints which must be taken into account.

Dolan-Nappi-Witten [22] also explained the appearance of spin chain Hamiltonian in the SYM side from the viewpoint of Yangian symmetry which is assumed to be related to the non-local charges found by BenaPolchinski-Roiban [9]. The classical/quantum integrabilities in string/gauge theories are expected to play an important role in a study of the AdS/CFT correspondence at non-BPS region.

The next step in a study of the classical integrability of AdS-superstring is to construct explicitly non-local charges by starting from a definite action of AdS-superstring. In this paper, we discuss a classical integrability in type IIB string theory on the $\mathrm{AdS}_{5} \times \mathrm{S}^{5}$ background. Firstly, we construct explicitly non-local charges, which are $\kappa$-invariant, by using the RoibanSiegel formulation [48] of superstring on the $\mathrm{AdS}_{5} \times \mathrm{S}^{5}$. This formulation is more suitable to discuss non-local charges rather than the Metsaev-Tseytlin formulation [43], since (1) the WZ term is bilinear of the left-invariant (LI) currents, (2) the global symmetry is realized linearly, and (3) the matrixvalued coordinates give simpler expression of the LI currents rather than using an exponential parameterization. Next, the super Yangian algebra is presented by calculating the Poisson bracket of the non-local charges. The resulting algebra represents a GL(4|4) super Yangian algebra. In that time the Serre relation is also shown. In addition, we discuss the classical integrability of the AdS-superstring theory in the Roiban-Siegel formulation by considering the Lax pair and transfer matrix. Following the standard 
discussion, the Poisson bracket of the transfer matrices gives a classical $r$ matrix, which satisfies the classical Yang-Baxter equation.

This paper is organized as follows: In Section 2, we briefly review the Roiban-Siegel formulation of superstring on the $\mathrm{AdS}_{5} \times \mathrm{S}^{5}$, and we obtain the conserved flat currents which are the right-invariant (RI) currents. In Section 3, we explicitly construct an infinite set of non-local charges. Then we discuss the Yangian algebra generated by the first two of them. The Serre relation is also discussed. Moreover, we show the Lax pair and transfer matrix of AdS-superstring, and then the classical integrability is discussed. Section 4 is devoted to a conclusion and discussions.

\section{Roiban-Siegel formulation of superstring on $\mathrm{AdS}_{5} \times \mathrm{S}^{5}$}

In this section, we will briefly review the Roiban-Siegel formulation [48] of the type IIB superstring on the $\mathrm{AdS}_{5} \times \mathrm{S}^{5}$ background.

In the well-known approach of Metsaev-Tseytlin to construct the action of the type IIB string on the $\mathrm{AdS}_{5} \times \mathrm{S}^{5}$, a coset superspace construction is used. In this framework, strings propagate on the superspace:

$$
\frac{\mathrm{PSU}(2,2 \mid 4)}{\mathrm{SO}(1,4) \times \mathrm{SO}(5)}
$$

which has as even part the $\mathrm{AdS}_{5} \times \mathrm{S}^{5}$ geometry. The exponential parameterization of the coset representation, however, is very complicated and seems to be unsuitable to study non-local charges or classical integrability in which we are interested in this paper.

On the other hand, the Roiban-Siegel formulation is appropriate to our consideration. The starting point of this formulation is to simplify the coset superspace with Wick rotations ${ }^{1}$ as follows:

$$
\frac{\mathrm{PSU}(2,2 \mid 4)}{\mathrm{SO}(1,4) \times \mathrm{SO}(5)} \longrightarrow \frac{\mathrm{PSL}(4 \mid 4)}{(\mathrm{Sp}(4))^{2}} \longrightarrow \frac{\mathrm{GL}(4 \mid 4)}{(\mathrm{Sp}(4) \times \mathrm{GL}(1))^{2}}
$$

The Lie-algebra identifications: $\mathrm{SU}(4) \cong \mathrm{SL}(4)$ and $\mathrm{SO}(5) \cong \mathrm{Sp}(4)$ lead to the first rewriting and the relaxation of both the $\mathrm{P}$ and the $\mathrm{S}$ constraints means the second one. The two GL(1)'s can be chosen to act separately on the upper-left and lower-right blocks. It may be possible to use the PSL(4|4) form rather than GL(4|4). The PSL(4|4), however, does not have the representation in $\operatorname{Mat}(4 \mid 4)$, and so it is still inconvenient.

\footnotetext{
${ }^{1}$ In the following discussion, the group theoretical features are only concerned, and hence the signature is not so important. In any case, we can Wick rotate back.
} 
The coset elements $Z_{M}{ }^{A}$, i.e.,

$$
Z_{M}^{A}=\left(\begin{array}{cc}
Z_{m}{ }^{a} & Z_{m}{ }^{\bar{a}} \\
Z_{\bar{m}}{ }^{a} & Z_{\bar{m}^{\bar{a}}}
\end{array}\right) \quad \in \frac{\mathrm{GL}(4 \mid 4)}{(\mathrm{Sp}(4) \times \mathrm{GL}(1))^{2}},
$$

transform in the defining representation of the superconformal group. The index $M$ is acted upon by the global coordinate transformation GL(4|4) while the local $(\mathrm{Sp}(4) \times \mathrm{GL}(1))^{2}$ (i.e., local Lorentz $(\mathrm{Sp}(4)$ 's) and dilatation (GL(1)'s) acts on the $A$ indices. The indices $m(a)$ and $\bar{m}(\bar{a})$ are GL(4)'s $(\mathrm{Sp}(4) \times \mathrm{GL}(1)$ 's) in the upper-left and lower-right, respectively.

The LI current

$$
\left(J_{\mu}^{L}\right)_{A}{ }^{B}=\left(Z^{-1}\right)_{A}{ }^{M} \partial_{\mu} Z_{M}^{B}=\left(\begin{array}{cc}
\left(\mathbf{J}_{\mu}\right)_{a}^{b} & \left(j_{\mu}\right)_{a}^{\bar{b}} \\
\left(\bar{j}_{\mu}\right)_{\bar{a}}{ }^{b} & \left(\overline{\mathbf{J}}_{\mu}\right)_{\bar{a}}
\end{array}\right)
$$

is decomposed into the coset part and the gauge potential part as usual (for example, see [1]). Before considering the decomposition, it is convenient to introduce the decomposition of an arbitrary matrix $M_{a b} \in \mathrm{GL}(4)$,

$$
M_{a b}=-\frac{1}{4} \Omega_{a b} M_{c}^{c}+M_{(a b)}+M_{\langle a b\rangle} \equiv-\frac{1}{4} \Omega \operatorname{tr} M+(M)+\langle M\rangle,
$$

into a trace part, symmetric part, and traceless-antisymmetric part. By using this decomposition of GL(4) part, the LI current $\left(J^{L}\right)_{A}^{B}$ is decomposed as follow:

The coset part : $\left(\mathbf{J}_{\mu}\right)^{\langle a b\rangle},\left(\overline{\mathbf{J}}_{\mu}\right)^{\langle\bar{a} \bar{b}\rangle},\left(j_{\mu}\right)^{a \bar{b}},\left(\bar{j}_{\mu}\right)^{\bar{a} b}$,

The gauge part : $\left(\mathbf{J}_{\mu}\right)^{(a b)},\left(\overline{\mathbf{J}}_{\mu}\right)^{(\bar{a} \bar{b})} \cdots \operatorname{Sp}(4)$ 's, $\operatorname{tr} \mathbf{J}, \operatorname{tr} \overline{\mathbf{J}} \cdots \mathrm{GL}(1)$ 's.

\subsection{Constraints and Hamiltonian}

The Roiban-Siegel action of AdS-superstring is given by these LI currents as

$$
\begin{aligned}
S= & \int d^{2} \sigma \mathcal{L}, \\
\mathcal{L}= & \frac{1}{2}\left\{-\sqrt{-g} g^{\mu \nu}\left(\mathbf{J}_{\mu}^{\langle a b\rangle} \mathbf{J}_{\langle a b\rangle \nu}-\overline{\mathbf{J}}_{\mu}^{\langle\bar{a} \bar{b}\rangle} \overline{\mathbf{J}}_{\nu\langle\bar{a} \bar{b}\rangle}\right)\right. \\
& \left.+\frac{k}{2} \epsilon^{\mu \nu}\left(E^{1 / 2} j_{\mu}^{a \bar{b}} j_{\nu a \bar{b}}-E^{-1 / 2} \bar{j}_{\mu}^{\bar{a} b} \bar{j}_{\nu \bar{a} b}\right)\right\},
\end{aligned}
$$

with $k= \pm 1$ and $E=\operatorname{sdet} Z_{M}{ }^{A}$. We follow the notation in [31] where canonical momentum for $Z_{M}{ }^{A}$ is defined as

$$
\Pi_{A}^{M}=\frac{\delta^{r} S}{\delta \dot{Z}_{M}^{A}}(-)^{A},
$$


and the canonical Poisson bracket is defined as

$$
\left[Z_{M}^{A}(\sigma), \Pi_{B}^{N}\left(\sigma^{\prime}\right)\right]_{\mathrm{P}}=(-1)^{A} \delta_{B}^{A} \delta_{M}^{N} \delta\left(\sigma-\sigma^{\prime}\right) .
$$

The Hamiltonian is given by

$$
\mathcal{H}=\int d \sigma\left[\sum_{M, A} \Pi_{A}^{M} \dot{Z}_{M}^{A}(-)^{A}-\mathcal{L}\right] .
$$

The supercovariant derivatives are

$$
D_{A}{ }^{B}=\Pi_{A}^{M} Z_{M}^{B}=\left(\begin{array}{cc}
\mathbf{D}_{a}^{b} & D_{a}^{\bar{b}} \\
\bar{D}_{\bar{a}}^{b} & \overline{\mathbf{D}}_{\bar{a}}^{\bar{b}}
\end{array}\right),
$$

generating right (local) GL(4|4) transformations

$$
\delta_{\Lambda(\sigma)} Z_{M}^{A}=\left[Z_{M}^{A}, \mathrm{~S} \operatorname{Tr} D \Lambda(\sigma)\right]=Z_{M}^{B} \Lambda_{B}{ }^{A}(\sigma) .
$$

Its subgroups $\mathrm{GL}(1), \mathrm{Sp}(4), \overline{\mathrm{GL}(1)}, \overline{\mathrm{Sp}(4)}$ are local gauge symmetries, and their generators are set to be constraints in our approach as follows:

$$
\operatorname{tr} \mathbf{D}=(\mathbf{D})=\operatorname{tr} \overline{\mathbf{D}}=(\overline{\mathbf{D}})=0 .
$$

These correspond to gauge degrees of freedom (2.3).

From the definition of the canonical momenta (2.5), we have following primary constraints

$$
\left\{\begin{array}{l}
F_{a}^{\bar{b}}=E^{1 / 4} D_{a}^{\bar{b}}+\frac{k}{2} E^{-1 / 4}\left(\bar{j}_{\sigma}\right)^{\bar{b}}{ }_{a}=0 \\
\bar{F}_{\bar{a}}{ }^{b}=E^{-1 / 4} \bar{D}_{\bar{a}}{ }^{b}+\frac{k}{2} E^{1 / 4}\left(j_{\sigma}\right)^{b}{ }_{\bar{a}}=0
\end{array} .\right.
$$

First class part of the fermionic constraints is

$$
\left\{\begin{array}{l}
B_{a}^{\bar{b}}=(\langle\mathbf{D}\rangle F+F\langle\overline{\mathbf{D}}\rangle)_{a}{ }^{\bar{b}}-\left(\left\langle\overline{\mathbf{J}}_{\sigma}\right\rangle \bar{F}+\bar{F}\left\langle\mathbf{J}_{\sigma}\right\rangle\right)^{\bar{b}}{ }_{a}=0 \\
\bar{B}_{\bar{a}}{ }^{b}=(\langle\overline{\mathbf{D}}\rangle \bar{F}+\bar{F}\langle\mathbf{D}\rangle)_{\bar{a}}{ }^{b}-\left(\left\langle\mathbf{J}_{\sigma}\right\rangle F+F\left\langle\overline{\mathbf{J}}_{\sigma}\right\rangle\right)^{b}{ }_{\bar{a}}=0
\end{array}\right.
$$

generating the kappa symmetry. The Hamiltonian is given by

$$
\begin{gathered}
\mathcal{H}=\int d \sigma\left[-\frac{2}{\sqrt{-g} g^{00}} A_{\perp}-\frac{2 g^{01}}{g^{00}} A_{\|}+\operatorname{tr}\{F \bar{\lambda}+\bar{F} \lambda\}\right] \\
\left\{\begin{array}{c}
A_{\perp}=\frac{1}{2} \operatorname{tr}\left[\langle\mathbf{D}\rangle^{2}+\left\langle\mathbf{J}_{\sigma}\right\rangle^{2}-\langle\overline{\mathbf{D}}\rangle^{2}-\left\langle\overline{\mathbf{J}}_{\sigma}\right\rangle^{2}\right]=0 \\
A_{\|}=\operatorname{tr}\left[\langle\mathbf{D}\rangle\left\langle\mathbf{J}_{\sigma}\right\rangle-\langle\overline{\mathbf{D}}\rangle\left\langle\overline{\mathbf{J}}_{\sigma}\right\rangle\right]=0
\end{array}\right.
\end{gathered}
$$

with multipliers $\lambda$ 's determined consistently

$$
\begin{aligned}
& \lambda=-\frac{2}{\sqrt{-g} g^{00}}\left(E^{-1 / 4} \bar{j}_{\sigma}\right)-\frac{2 g^{01}}{g^{00}}\left(-E^{1 / 4} j_{\sigma}\right) \\
& \bar{\lambda}=-\frac{2}{\sqrt{-g} g^{00}}\left(-E^{1 / 4} j_{\sigma}\right)-\frac{2 g^{01}}{g^{00}}\left(E^{-1 / 4} \bar{j}_{\sigma}\right)
\end{aligned} .
$$


In the Hamiltonian (2.12) multipliers of the other first class constraints, the local GL(1), $\mathrm{Sp}(4), \overline{\mathrm{GL}(1)}, \overline{\mathrm{Sp}(4)}$ constraints in $(2.9)$ and $B=\bar{B}=0$ in (2.11), are set to be zero.

Time development is determined by the Hamiltonian (2.12), where we take the conformal gauge $g_{00}+g_{11}=0=g_{01}$ as well as $E=1$ gauge,

$$
\begin{gathered}
\mathcal{H}=-\int d \sigma \operatorname{tr}\left[\frac{1}{2}\left\{\langle\mathbf{D}\rangle^{2}+\left\langle\mathbf{J}_{\sigma}\right\rangle^{2}-\langle\overline{\mathbf{D}}\rangle^{2}-\left\langle\overline{\mathbf{J}}_{\sigma}\right\rangle^{2}\right\}\right. \\
\left.+\left(\bar{D} \bar{j}_{\sigma}-D j_{\sigma}+k j_{\sigma} \bar{j}_{\sigma}\right)\right] .
\end{gathered}
$$

The equation of motion is given as

$$
\begin{aligned}
\dot{Z}_{M}{ }^{A} & =\left[Z_{M}{ }^{A}, \mathcal{H}\right]=Z_{M}{ }^{B} \Gamma_{B}{ }^{A}, \\
\Gamma_{B}{ }^{A} & =\left(\begin{array}{cc}
\langle\mathbf{D}\rangle & -\bar{j}_{\sigma} \\
-j_{\sigma} & \langle\overline{\mathbf{D}}\rangle
\end{array}\right) \approx\left(\begin{array}{cc}
\langle\mathbf{D}\rangle & 2 k D \\
2 k \bar{D} & \langle\overline{\mathbf{D}}\rangle
\end{array}\right),
\end{aligned}
$$

where $\approx$ denotes that the fermionic constraints (2.10) are used.

In order to construct explicitly an infinite set of conserved non-local charges, we will consider LI and RI currents below.

\subsection{LI currents}

The LI currents (2.1) in the conformal gauge are given as

$$
J_{\mu}^{L}=Z^{-1} \partial_{\mu} Z=\left\{\begin{array}{l}
\left(J_{\tau}^{L}\right)_{A}{ }^{B}=\Gamma_{A}^{B} \\
\left(J_{\sigma}^{L}\right)_{A} B=\left(Z^{-1} \partial_{\sigma} Z\right)_{A}{ }^{B}
\end{array}\right.
$$

with $\Gamma_{A}^{B}$ in (2.16). They satisfy the flatness condition by definition

$$
\epsilon^{\mu \nu} \partial_{\mu} J_{\nu}^{L}=-\epsilon^{\mu \nu} Z^{-1} \partial_{\mu} Z Z^{-1} \partial_{\nu} Z=-\epsilon^{\mu \nu} J_{\mu}^{L} J_{\nu}^{L} .
$$

The equation of motion for the LI currents is calculated by taking commutators with the Hamiltonian (2.15)

$$
\begin{gathered}
\partial_{\tau} J_{\tau}^{L}=\nabla_{\sigma} J_{\sigma}^{L}+2(2-k)\left[\left(\begin{array}{ll}
\langle\mathbf{D}\rangle & \\
& \langle\overline{\mathbf{D}}\rangle
\end{array}\right),\left(\begin{array}{cc}
\bar{D} & D
\end{array}\right)\right] \\
-(2 k-1)\left[\left(\begin{array}{cc}
\left\langle\mathbf{J}_{\sigma}\right\rangle & \\
& \left\langle\overline{\mathbf{J}}_{\sigma}\right\rangle
\end{array}\right),\left(\begin{array}{ll}
\bar{j}_{\sigma} & j_{\sigma}
\end{array}\right)\right]
\end{gathered}
$$

with the covariant derivatives on the LI currents:

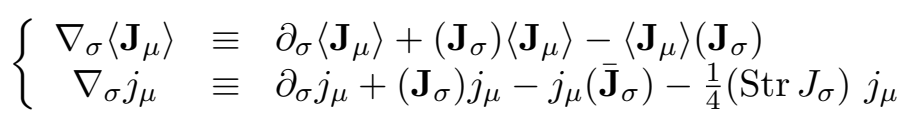

and similar expression holds for the barred sector. 


\section{$2.3 \quad$ RI currents}

The RI currents are generators of the left multiplication on a coset element $Z_{M}{ }^{A}$ which is the global GL(4|4) symmetry $\delta_{\Lambda} Z_{M}{ }^{A}=\left[Z_{M}{ }^{A}, \int \operatorname{STr} \Lambda Z \Pi\right]_{\mathrm{P}}=$ $\Lambda_{M}{ }^{N} Z_{N}{ }^{A}$. Since the action (2.4) is written in terms of the LI currents, which are manifestly invariant under the left multiplication, the RI currents must be conserved. The generator of the left multiplication is the integration of the $\tau$-component of the RI currents

$$
\left(J_{\tau}^{R}\right)_{M}^{N}=Z_{M}^{A} \Pi_{A}^{N}=Z_{M}^{A} D_{A}^{B}\left(Z^{-1}\right)_{B}{ }^{N} .
$$

The $\sigma$-component of the RI currents begins with $\left(\partial_{\sigma} Z\right) Z^{-1}$ which is neither invariant nor covariant under the right action on $Z_{M}{ }^{A}$, that is the local $[\mathrm{Sp}(4) \times \mathrm{GL}(1)]^{2}$. Then we covariantize these local symmetries:

$$
\begin{aligned}
\left(\partial_{\sigma} Z\right) Z^{-1} \longrightarrow\left(J_{\sigma}^{R}\right)_{M^{N}} & \equiv\left(\Delta_{\sigma} Z\right) Z^{-1} \equiv\left(\partial_{\sigma} Z\right) Z^{-1}+Z \mathcal{A} Z^{-1} \\
& =Z_{M}{ }^{A}\left(J_{\sigma}^{L}+\mathcal{A}\right)_{A}{ }^{B}\left(Z^{-1}\right)_{B}{ }^{N}
\end{aligned}
$$

by introducing the $[\mathrm{Sp}(4) \otimes \mathrm{GL}(1)]^{2}$-covariant derivative. The bosonic part of the gauge connection is obtained from the one on the LI currents (2.20) as follows:

$$
\left\{\begin{array}{l}
\mathcal{A}^{a b}=-\left(\mathbf{J}_{\sigma}\right)^{(a b)}+\frac{1}{4} \Omega^{a b} \operatorname{tr} \mathbf{J}_{\sigma} \\
\mathcal{A}^{\bar{a} \bar{b}}=-\left(\overline{\mathbf{J}}_{\sigma}\right)^{(\bar{a} \bar{b})}+\frac{1}{4} \Omega^{\bar{a} \bar{b}} \operatorname{tr} \overline{\mathbf{J}}_{\sigma}
\end{array} .\right.
$$

Then the $\sigma$-component of the covariant RI current is invariant under the local symmetry transformation $Z \rightarrow Z h^{-1}$ with $h \in[\mathrm{Sp}(4) \otimes \mathrm{GL}(1)]^{2}$ by the gauge field transformation as $\mathcal{A}+\left(\partial_{\mu} h^{-1}\right) h \rightarrow \mathcal{A}$. Bosonic part of the gauge connection $\mathcal{A}$ is an element of the stability group.

The current only with (2.23) is however not conserved. For a superstring, the existence of the fermionic constraints requires the non-zero fermionic elements of $\mathcal{A}$. This fact is related to that the super-coset space is not a symmetric space. We determine the fermionic components of the gauge connection $\mathcal{A}$ from the current conservation law,

$$
\partial^{\mu} J_{\mu}^{R}=-\partial_{\tau} J_{\tau}^{R}+\partial_{\sigma} J_{\sigma}^{R}=0 .
$$

The first term in $(2.24)$ is

$$
\begin{aligned}
\partial_{\tau} J_{\tau}^{R}=Z\left\{\partial_{\sigma}\left(\begin{array}{cc}
\left\langle\mathbf{J}_{\sigma}\right\rangle & \frac{k}{2} j_{\sigma} \\
\frac{k}{2} \bar{j}_{\sigma} & \left\langle\overline{\mathbf{J}}_{\sigma}\right\rangle
\end{array}\right)+\left[J_{\sigma}^{L},\left(\begin{array}{cc}
\left\langle\mathbf{J}_{\sigma}\right\rangle & \frac{k}{2} j_{\sigma} \\
\frac{k}{2} \bar{j}_{\sigma} & \left\langle\overline{\mathbf{J}}_{\sigma}\right\rangle
\end{array}\right)\right]\right. \\
\left.+2(k-1)\left[\left(\begin{array}{cc}
\langle\mathbf{D}\rangle & \\
& \langle\overline{\mathbf{D}}\rangle
\end{array}\right),\left(\begin{array}{cc}
\bar{D} & D
\end{array}\right)\right]\right\} Z^{-1} .
\end{aligned}
$$


The second term in $(2.24)$ is

$$
\partial_{\sigma} J_{\sigma}^{R}=Z\left\{\partial_{\sigma}\left(J_{\sigma}^{L}+\mathcal{A}\right)+\left[J_{\sigma}^{L}, \mathcal{A}\right]\right\} Z^{-1} .
$$

Then we can read off

$$
J_{\sigma}^{L}+\mathcal{A}=\left(\begin{array}{cc}
\left\langle\mathbf{J}_{\sigma}\right\rangle & \frac{k}{2} j_{\sigma} \\
\frac{k}{2} \bar{j}_{\sigma} & \left\langle\overline{\mathbf{J}}_{\sigma}\right\rangle
\end{array}\right) \equiv\left\langle J_{\sigma}^{L}\right\rangle,
$$

and the $\kappa$-parameter $k=1$ is chosen. The fermionic component of the connection is determined as

$$
\left\{\begin{array}{l}
\mathcal{A}^{a \bar{b}}=\left(\frac{k}{2}-1\right) j_{\sigma}^{a \bar{b}} \\
\mathcal{A}^{\bar{a} b}=\left(\frac{k}{2}-1\right) \bar{j}_{\sigma}^{\bar{a} b}
\end{array}\right.
$$

Next we examine the flatness for the RI currents. By using the equation of motions

$$
\partial_{\tau}\left\langle J_{\sigma}^{L}\right\rangle=\partial_{\sigma} D+[D, \mathcal{A}]-\left[\left(\begin{array}{cc} 
& D \\
\bar{D} &
\end{array}\right),\left\langle J_{\sigma}^{L}\right\rangle\right]
$$

the flatness is given as

$$
\begin{aligned}
\partial_{\tau} J_{\sigma}^{R}-\partial_{\sigma} J_{\tau}^{R} & =Z\left\{\partial_{\tau}\left\langle J_{\sigma}^{L}\right\rangle+\left[\Gamma,\left\langle J_{\sigma}^{L}\right\rangle\right]-\partial_{\sigma} D-\left[J_{\sigma}^{L}, D\right]\right\} Z^{-1} \\
& =2 Z\left\{\left[D,\left\langle J_{\sigma}^{L}\right\rangle\right]\right\} Z^{-1} \\
& =2\left(J_{\tau}^{R} J_{\sigma}^{R}-J_{\sigma}^{R} J_{\tau}^{R}\right) .
\end{aligned}
$$

The front factor 2 can be absorbed by rescaling $J^{R} \rightarrow(1 / 2) J^{R}$, reproducing the usual flatness condition $d J^{R}=J^{R} J^{R}$.

In the next section, we will construct an infinite set of conserved non-local charges by using the RI currents.

\section{Super Yangian and classical integrability of AdS-string}

In this section, we will discuss super Yangian algebra of type IIB superstring on the $\mathrm{AdS}_{5} \times S^{5}$. We will construct an infinite set of non-local charges in the canonical formalism and calculate the Poisson bracket algebra of them. The resulting algebra is a Hopf-Poisson algebra called the Yangian algebra. We can show the Yangian algebra in terms of the supermatrix, i.e., the super Yangian algebra of the AdS-superstring. To begin with, we explicitly construct non-local charges. Then the Yangian algebra is calculated by using the Poisson bracket. The Serre relation is also satisfied. In addition, the Lax 
pair and the transfer matrix are presented, the existence of them implies a classical integrability of AdS-superstring.

\subsection{Construction of non-local charges}

Here we shall construct non-local charges following Brezin et al. [15]. In previous section, we have obtained the conserved and flat current which is the RI currents

$$
\left(J^{R}\right)_{\mu}=\left\{\begin{array}{l}
J_{\tau}^{R}=Z \Pi \\
J_{\sigma}^{R}=\left(\partial_{\sigma} Z\right) Z^{-1}+Z \mathcal{A} Z^{-1}=Z\left(J_{\sigma}^{L}+\mathcal{A}\right) Z^{-1}
\end{array}\right.
$$

with

$$
J_{\sigma}^{L}+\mathcal{A}=\left(\begin{array}{cc}
\left\langle\mathbf{J}_{\sigma}\right\rangle & \frac{1}{2} j_{\sigma} \\
\frac{1}{2} \bar{j}_{\sigma} & \left\langle\overline{\mathbf{J}}_{\sigma}\right\rangle
\end{array}\right) .
$$

By defining the covariant derivative

$$
\mathcal{D}_{\mu}=\partial_{\mu}-2 J_{\mu}^{R}
$$

the flatness condition (2.26) and the conservation law are expressed as

$$
\epsilon^{\mu \nu}\left[\mathcal{D}_{\mu}, \mathcal{D}_{\nu}\right]=0, \quad\left[\partial^{\mu}, \mathcal{D}_{\mu}\right]=0 .
$$

Conserved non-local currents are constructed as follows: Beginning with a conserved current

$$
\left(\mathcal{J}_{n}\right)_{\mu}=\epsilon_{\mu \nu} \partial^{\nu} \chi_{n} \quad(n \geq 0),
$$

another conserved current can be constructed as

$$
\left(\mathcal{J}_{n+1}\right)_{\mu}=\mathcal{D}_{\mu} \chi_{n}
$$

where equations in $(3.1)$ are used. We take $\left(\mathcal{J}_{-1}\right)_{\mu}=0$ and $\chi_{-1}=-\frac{1}{2}$, then the conserved non-local currents are given as

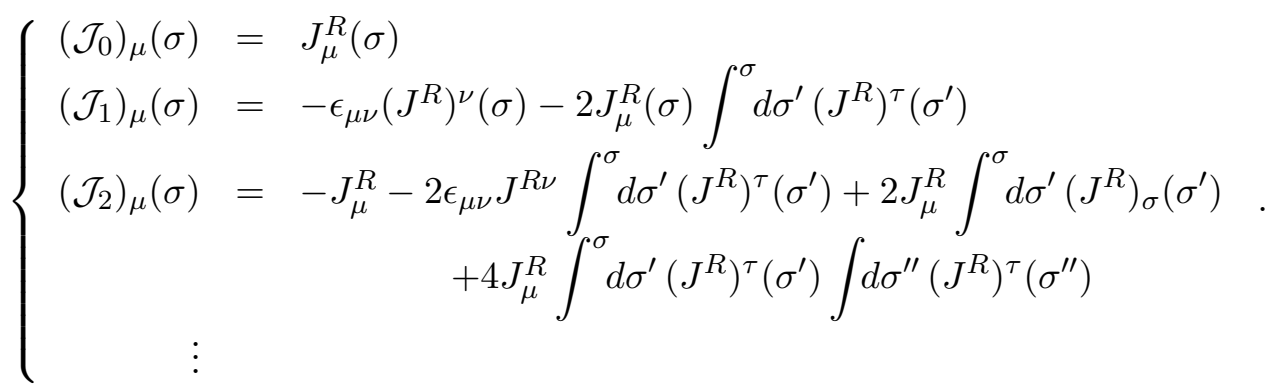

There exists infinite number of the conserved non-local charges $Q_{n}$ which are the $\sigma$ integration of the $\tau$-component of the non-local currents, $\int d \sigma\left(\mathcal{J}_{n}\right)_{\tau}$. 
It is commented about the range of the spatial direction we consider. In studies of Yangian of sigma models such as chiral principle models and $\mathrm{O}(N)$ sigma models, the integral for the spatial direction is performed from $-\infty$ to $+\infty$. But we would like to consider a closed string satisfying a periodic boundary condition ${ }^{2}$ as

$$
Z_{M}^{A}(\tau, \sigma)=Z_{M}^{A}(\tau, \sigma+2 \pi)
$$

with $-\infty \leq \tau \leq \infty$ and $0 \leq \sigma \leq 2 \pi$ which are the coordinates of a cylinder. We naively take this condition ${ }^{3}$, but then the non-abelian generators may be broken by finite size effects.

Hence the super Yangian generators ${ }^{4}$ are given by

$$
\begin{aligned}
Q_{0} & \equiv \int_{0}^{2 \pi} d \sigma\left(\mathcal{J}_{0}\right)_{\tau}=\int_{0}^{2 \pi} d \sigma J_{\tau}^{R}(\sigma), \\
Q_{1} & \equiv-\int_{0}^{2 \pi} d \sigma\left(\mathcal{J}_{1}\right)_{\tau}=\int_{0}^{2 \pi} d \sigma J_{\sigma}^{R}(\sigma)-2 \int_{0}^{2 \pi} d \sigma \int_{0}^{\sigma} d \sigma^{\prime}\left[J_{\tau}^{R}(\sigma), J_{\tau}^{R}\left(\sigma^{\prime}\right)\right] \\
& =\int_{0}^{2 \pi} d \sigma J_{\sigma}^{R}(\sigma)-\int_{0}^{2 \pi} d \sigma \int_{0}^{2 \pi} d \sigma^{\prime} \epsilon\left(\sigma-\sigma^{\prime}\right)\left[J_{\tau}^{R}(\sigma), J_{\tau}^{R}\left(\sigma^{\prime}\right)\right],
\end{aligned}
$$

where we have introduced the function $\epsilon(x)=\theta(x)-\theta(-x)$, so that

$$
\epsilon(x)=1 \quad(x>0), \quad \epsilon(-x)=-1 \quad(x<0) .
$$

We will calculate the Poisson bracket algebra of the charges $Q_{0}$ and $Q_{1}$ below. The $\kappa$-invariance and the gauge invariances are confirmed explicitly by the Poisson bracket between the charges and the gauge generators which

\footnotetext{
${ }^{2}$ The classical Poisson bracket may be inconsistent with non-trivial boundary conditions when the current algebra includes non-ultra local terms [20]. As an example, the boundary condition $\left.X_{a}(x)\right|_{ \pm \infty}=$ const., i.e., $\left.\partial_{x} X_{a}(x)\right|_{ \pm \infty}=0$, is incompatible with the definition of the Poisson bracket at the boundary as follows:

$$
\left[X_{a}(x), P^{b}(y)\right]_{\mathrm{P}}=\left.\delta_{a}^{b} \delta(x-y) \rightarrow\left[\partial_{x} X_{a}(x), P^{b}(y)\right]_{\mathrm{P}}\right|_{x= \pm \infty}=0 \neq\left.\delta_{a}^{b} \partial_{x} \delta(x-y)\right|_{x= \pm \infty} .
$$

The modification of the Poisson bracket implies to change the vacua in the theory [50]. This view is proposed in [26] and examined in [36].

${ }^{3}$ In the case of the finite interval $[0,2 \pi]$, we should possibly modify the $J^{R}$, for example, as follows:

$$
\begin{aligned}
& \left(J^{R}\right)_{\sigma}(\tau, \sigma)=\partial_{\tau} \chi_{0}(\tau, \sigma), \\
& \chi_{0}(\tau, \sigma)=\int_{0}^{\sigma} d \sigma^{\prime}\left(J^{R}\right)_{\tau}\left(\tau, \sigma^{\prime}\right)+\int_{-\infty}^{\tau} d \tau^{\prime}\left(J^{R}\right)_{\sigma}\left(\tau^{\prime}, \sigma=0\right) .
\end{aligned}
$$

The non-local charge $Q_{1}$ constructed from the above currents may be conserved and satisfy (3.6). However, the cubic Serre relation of the Yangian and (3.6) may contain additional terms and so we need some efforts to justify (3.4). In this paper we present the computation without the extra terms in (3.4) as the first step.

${ }^{4}$ Non-local charges and local charges in supersymmetric $\mathrm{NLSM}_{2}$ are discussed in $[17,25]$. But the supersymmetries are not space-time but world-sheet.
} 
are LI currents. Firstly, we will consider the bosonic case to recollect the (bosonic) Yangian algebra. After the discussion of the bosonic case, we will consider the supersymmetric case by taking care of the gradation of fermionic variables.

\subsection{Bosonic Yangian algebra}

Before going to the full supersymmetric case, let us consider a bosonic part, which is described like a principle chiral model, contained in the RoibanSiegel AdS-superstring. For simplicity, we will concentrate on the upper left-part $\left(\mathrm{AdS}_{5}\right)$.

The Poisson bracket of a bosonic (upper-left) part is given by

$$
\left[\mathbf{z}_{m}^{a}(\sigma), \pi_{b}^{n}\left(\sigma^{\prime}\right)\right]_{\mathrm{P}}=\delta_{m}^{n} \delta_{b}^{a} \delta\left(\sigma-\sigma^{\prime}\right)
$$

where the "delta function" has a periodicity with $2 \pi$. The bosonic RI currents are

$$
\left(J^{R}\right)_{\mu}=\left\{\begin{aligned}
\left(J_{\tau}^{R}\right)_{m}{ }^{n} & =\mathbf{z}_{m}{ }^{a} \pi_{a}{ }^{n} \\
\left(J_{\sigma}^{R}\right)_{m}{ }^{n} & =\left(\partial_{\sigma} \mathbf{z}\right)_{m}{ }^{a}\left(\mathbf{z}^{-1}\right)_{a}{ }^{n}+\mathbf{z}_{m}{ }^{a} \mathcal{A}_{a}{ }^{b}\left(\mathbf{z}^{-1}\right)_{b}{ }^{n} \\
& =(\mathbf{z})_{m}{ }^{a}\left(J_{\sigma}^{L}+\mathcal{A}\right)_{a}{ }^{b}\left(\mathbf{z}^{-1}\right)_{b}{ }^{n}
\end{aligned}\right.
$$

with $\mathbf{z}_{m}{ }^{a}\left(\mathbf{z}^{-1}\right)_{a}{ }^{n}=\delta_{m}^{n}$. In the calculation of Yangian algebra, it is convenient to use the Poisson bracket in terms of $J_{\tau m}^{R}{ }^{n}$ and $J_{\sigma m}^{R}{ }^{n}$ :

$$
\begin{aligned}
{\left[\int\left(J_{\tau}^{R}\right)_{m}^{n}(\sigma),\left(J_{\tau}^{R}\right)_{l}^{k}\left(\sigma^{\prime}\right)\right]_{\mathrm{P}} } & =\left(\delta_{m}^{k}\left(J_{\tau}^{R}\right)_{l}^{n}-\delta_{l}^{n}\left(J_{\tau}^{R}\right)_{m}^{k}\right)\left(\sigma^{\prime}\right), \\
{\left[\int\left(J_{\tau}^{R}\right)_{m}^{n}(\sigma),\left(J_{\sigma}^{R}\right)_{l}^{k}\left(\sigma^{\prime}\right)\right]_{\mathrm{P}} } & =\left(\delta_{m}^{k}\left(J_{\sigma}^{R}\right)_{l}{ }^{n}-\delta_{l}^{n}\left(J_{\sigma}^{R}\right)_{m}^{k}\right)\left(\sigma^{\prime}\right) \\
{\left[\left(J_{\sigma}^{R}\right)_{m}^{n}(\sigma),\left(J_{\sigma}^{R}\right)_{l}{ }^{k}\left(\sigma^{\prime}\right)\right]_{\mathrm{P}} } & =0 .
\end{aligned}
$$

It is denoted that $\left[\int J_{\mu}^{R}, \mathcal{A}\right]=0$ because the gauge connection $\mathcal{A}$ is written in terms of the LI currents, $\left[\int\left(J_{\mu}^{R}\right)_{m}^{n},\left(J_{\nu}^{L}\right)_{a}^{b}\right]=0$. As a remark, we have to be careful to treat the non-ultra local term in the Poisson bracket of $J_{\tau}^{R}$ and $J_{\sigma}^{R}$, as in the principle chiral models. For the treatment for the non-ultra local term, see Appendix B.

We obtain the Yangian algebra:

$$
\begin{aligned}
& {\left[Q_{0 m}^{n}, Q_{0 l}^{k}\right]_{\mathrm{P}}=\delta_{m}^{k} Q_{0 l}^{n}-\delta_{l}^{n} Q_{0 m}^{k},} \\
& {\left[Q_{0 m}^{n}, Q_{1 l}^{k}\right]_{\mathrm{P}}=\delta_{m}^{k} Q_{1 l}^{n}-\delta_{l}^{n} Q_{1 m}^{k},} \\
& {\left[Q_{1 m}^{n}, Q_{1 l}^{k}\right]_{\mathrm{P}}=\delta_{m}^{k} Q_{2 l}^{n}-\delta_{l}^{n} Q_{2 m}^{k}+4\left(Q_{0 m}^{k} Q_{0 l}^{p} Q_{0 p}^{n}-Q_{0 l}^{n} Q_{0 m}^{p} Q_{0 p}^{k}\right),}
\end{aligned}
$$


where we have defined a tri-local charge $Q_{2}$ as

$$
\begin{aligned}
Q_{2 m}^{k}(\tau) \equiv & -4 Q_{0 m}^{k}+2 \int_{0}^{2 \pi} d \sigma \int_{0}^{2 \pi} d \sigma^{\prime} \epsilon\left(\sigma-\sigma^{\prime}\right)\left[J_{\tau}^{R}(\sigma), J_{\sigma}^{R}\left(\sigma^{\prime}\right)\right]_{m}^{k} \\
& +4 \int_{0}^{2 \pi} d \sigma \int_{0}^{2 \pi} d \sigma^{\prime} \int_{0}^{2 \pi} d \sigma^{\prime \prime} \epsilon\left(\sigma-\sigma^{\prime}\right) \epsilon\left(\sigma^{\prime}-\sigma^{\prime \prime}\right) \\
& \times\left(J_{\tau}^{R}\right)_{m}^{p}(\sigma)\left(J_{\tau}^{R}\right)_{p}^{p^{\prime}}\left(\sigma^{\prime}\right)\left(J_{\tau}^{R}\right)_{p^{\prime}}^{k}\left(\sigma^{\prime \prime}\right) .
\end{aligned}
$$

In the calculation of the algebra, it is convenient to use the identity:

$$
\epsilon\left(\sigma^{\prime}-\sigma\right) \epsilon\left(\sigma-\sigma^{\prime \prime}\right)+\epsilon\left(\sigma-\sigma^{\prime}\right) \epsilon\left(\sigma^{\prime}-\sigma^{\prime \prime}\right)+\epsilon\left(\sigma-\sigma^{\prime \prime}\right) \epsilon\left(\sigma^{\prime \prime}-\sigma^{\prime}\right)=-1 .
$$

The Serre relation, which is needed to ensure the integrable structure is the standard Yangian algebra, is shown, up to the ambiguity of linear term with respect to $Q_{0}$, as follows:

$$
\begin{aligned}
& {\left[Q_{0 m}^{n},\left[Q_{1 l}^{k}, Q_{1 p}^{q}\right]_{\mathrm{P}}\right]_{\mathrm{P}}-\left[Q_{1 m}^{n},\left[Q_{0 l}^{k}, Q_{1 p}^{q}\right]_{\mathrm{P}}\right]_{\mathrm{P}}} \\
& \quad=4\left[Q_{0 m}^{n},\left[Q_{0 l}^{p^{\prime}} Q_{0 p^{\prime}}^{k}, Q_{0 p}^{p^{\prime \prime}} Q_{0 p^{\prime \prime}}^{q}\right]_{\mathrm{P}}\right]_{\mathrm{P}}-4\left[Q_{0 m}^{p^{\prime}} Q_{0 p^{\prime}}^{n},\left[Q_{0 l}^{k}, Q_{0 p}^{p^{\prime \prime}} Q_{0 p^{\prime \prime}}^{q}\right]_{\mathrm{P}}\right]_{\mathrm{P}}
\end{aligned}
$$

The point is that the higher order charge $Q_{2}$, which appears in the $\left[Q_{1}, Q_{1}\right]$, disappears in the above Serre relation. The fifth linear term in $Q_{0}$ becomes the tri-linear in $Q_{0}{ }^{5}$.

In the next subsection, we will consider the full supermatrix case by following the above-mentioned regularization and order of limits.

\subsection{Super Yangian algebra}

Let now us calculate the Poisson bracket algebra of non-local charges in the supersymmetric case and show the super Yangian algebra.

\footnotetext{
${ }^{5}$ It is shown in the adjoint index of the matrix as

$$
\left[Q_{1}^{a},\left[Q_{1}^{b}, Q_{0}^{c}\right]\right]+\text { cyclic in } a, b, c=12 f^{a r l} f^{b s k} f^{c t m} f^{t r s} Q_{0}^{l} Q_{0}^{m} Q_{0}^{k},
$$

where $f^{a b c}$ with $\left[Q_{0}^{a}, Q_{0}^{b}\right]=f^{a b c} Q_{0}^{c}$ is the totally antisymmetric structure constant for the $\mathrm{SL}(4)$ group after the GL(1) gauge fixed.
} 
Using the Poisson bracket (2.6), the current algebra in terms of the supermatrix is given by

$$
\begin{aligned}
& {\left[\int\left(J_{\tau}^{R}\right)_{M}^{N}(\sigma),\left(J_{\tau}^{R}\right)_{L}^{K}\left(\sigma^{\prime}\right)\right\}_{\mathrm{P}}} \\
& \quad=(-)^{N}\left[(-)^{(N+L)(1+M+L)} \delta_{M}^{K}\left(J_{\tau}^{R}\right)_{L}^{N}-\delta_{L}^{N}\left(J_{\tau}^{R}\right)_{M}^{K}\right]\left(\sigma^{\prime}\right), \\
& {\left[\int\left(J_{\tau}^{R}\right)_{M}^{N}(\sigma),\left(J_{\sigma}^{R}\right)_{L}^{K}\left(\sigma^{\prime}\right)\right\}_{\mathrm{P}}} \\
& \quad=(-)^{N}\left[(-)^{(N+L)(1+M+L)} \delta_{M}^{K} J_{\sigma}^{R}{ }^{N}-\delta_{L}^{N} J_{\sigma}^{R}{ }^{K}\right]\left(\sigma^{\prime}\right) .
\end{aligned}
$$

For the super case, the fermionic constraints (2.10) exist. Half of it are first-class constraints generating the $\kappa$-symmetry [31] as the same situation of the flat superstring, and the non-local charges commute with it since the fermionic constraints are LI currents, $\left\{\int J_{\mu}^{R}, J_{\nu}^{L}\right\}=0$. Another half constraints are second-class constraints which require to modify the Poisson bracket to the Dirac bracket: $\{,\}_{\mathrm{P}} \rightarrow\{,\}_{\text {Dirac }}$. Fortunately, the Dirac brackets of the RI currents are equal to the Poisson bracket, $\left\{\int J_{\mu}^{R}, *\right\}_{\text {Dirac }}=$ $\left\{\int J_{\mu}^{R}, *\right\}_{\mathrm{P}}$, since the fermionic second-class constraints are also LI currents. Non-ultra local terms also appear in the supersymmetric case, but the prescription is the same as in the bosonic case.

The super Yangian algebra i.e., the Poisson bracket algebra of non-local charges in the case of supermatrix, is given by

$$
\begin{aligned}
{\left[Q_{0 M}^{N}, Q_{0 L}^{K}\right\}_{\mathrm{P}}=} & (-)^{N}\left[(-)^{(N+L)(1+M+L)} \delta_{M}^{K} Q_{0 L}^{N}-\delta_{L}^{N} Q_{0 M}^{K}\right] \\
{\left[Q_{0 M}^{N}, Q_{1 L}^{K}\right\}_{\mathrm{P}}=} & (-)^{N}\left[(-)^{(N+L)(1+M+L)} \delta_{M}^{K} Q_{1 L}^{N}-\delta_{L}^{N} Q_{1 M}^{K}\right] \\
{\left[Q_{1 M}^{N}, Q_{1 L}^{K}\right\}_{\mathrm{P}}=} & (-)^{N}\left[(-)^{(N+L)(1+M+L)} \delta_{M}^{K} Q_{2 L}^{N}-\delta_{L}^{N} Q_{2 M}^{K}\right] \\
& +(-)^{N}(-)^{(N+L)(1+M+L)} 4\left[Q_{0 L}^{P} Q_{0 P}^{N} Q_{0 M}^{K}-Q_{0 L}^{N} Q_{0 M}{ }^{P} Q_{0 P}^{K}\right]
\end{aligned}
$$

where we have defined $Q_{2}$ as

$$
\begin{aligned}
Q_{2 M}^{K}(\tau) \equiv & -4 Q_{0 M}^{K}+2 \int_{0}^{2 \pi} d \sigma \int_{0}^{2 \pi} d \sigma^{\prime} \epsilon\left(\sigma-\sigma^{\prime}\right)\left[\left(J_{\tau}^{R}\right)(\sigma),\left(J_{\sigma}^{R}\right)\left(\sigma^{\prime}\right)\right]_{M}^{K} \\
& +4 \int_{0}^{2 \pi} d \sigma \int_{0}^{2 \pi} d \sigma^{\prime} \int_{0}^{2 \pi} d \sigma^{\prime \prime} \epsilon\left(\sigma-\sigma^{\prime}\right) \epsilon\left(\sigma^{\prime}-\sigma^{\prime \prime}\right) \\
& \times\left(J_{\tau}^{R}\right)_{M}^{P}(\sigma)\left(J_{\tau}^{R}\right)_{P}^{P^{\prime}}\left(\sigma^{\prime}\right)\left(J_{\tau}^{R}\right)_{P^{\prime}}^{K}\left(\sigma^{\prime \prime}\right) .
\end{aligned}
$$

When we introduce the notation, following [16],

$$
\widehat{Q}_{i M}^{N} \equiv(-)^{M} Q_{i M}^{N} \quad(i=0,1,2, \ldots,),
$$


the super Yangian algebra is rewritten as follows:

$$
\begin{aligned}
{\left[\widehat{Q}_{0 M}^{N}, \widehat{Q}_{0 L}^{K}\right\}_{\mathrm{P}}=} & (-)^{(M+N)(L+K)} \delta_{M}^{K} \widehat{Q}_{0 L}^{N}-\delta_{L}^{N} \widehat{Q}_{0 M}^{K} \\
{\left[\widehat{Q}_{0 M}^{N}, \widehat{Q}_{1 L}^{K}\right\}_{\mathrm{P}}=} & (-)^{(M+N)(L+K)} \delta_{M}^{K} \widehat{Q}_{1 L}^{N}-\delta_{L}^{N} \widehat{Q}_{1 M}^{K} \\
{\left[\widehat{Q}_{1 M}^{N}, \widehat{Q}_{1 L}^{K}\right\}_{\mathrm{P}}=} & (-)^{(M+N)(L+K)} \delta_{M}^{K} \widehat{Q}_{2 L}^{N}-\delta_{L}^{N} \widehat{Q}_{2 M}^{K} \\
& +(-)^{(M+N)(L+K)} 4\left[\widehat{Q}_{0 L}^{P} \widehat{Q}_{0 P}^{N} \widehat{Q}_{0 M}^{K}-\widehat{Q}_{0 L}^{N} \widehat{Q}_{0 M}^{P} \widehat{Q}_{0 P}^{K}\right]
\end{aligned}
$$

This form is the familiar expression of the GL(4|4) ${ }^{6}$ super Yangian algebra (often denoted as $Y(\operatorname{gl}(4 \mid 4))$ or simply $Y(4 \mid 4))$. The super Yangian for GL $(M \mid N)$ was first defined by Nazarov [45]. It can be obtained as the generalization of the construction for the Yangian $Y(\operatorname{gl}(M))$ (bosonic case), based on the Lie algebra $\operatorname{gl}(M)$, to the case of the super Lie algebra $\operatorname{gl}(M \mid N)$. Its representations are studied by Zhang [52].

In addition, we can show the super Serre relation in the full supermatrix case:

$$
\begin{aligned}
& {\left[Q_{0 M}^{N},\left[Q_{1 L}^{K}, Q_{1 P}^{Q}\right\}_{\mathrm{P}}\right\}_{\mathrm{P}}-\left[Q_{1 M}^{N},\left[Q_{0 L}^{K}, Q_{1 P}^{Q}\right\}_{\mathrm{P}}\right\}_{\mathrm{P}}} \\
& \quad=\left[Q_{0 M}^{N},\left[Q_{0 L}^{P^{\prime}} Q_{0 P^{\prime}}^{K}, Q_{0 P}^{P^{\prime \prime}} Q_{0 P^{\prime \prime}}^{Q}\right\}_{\mathrm{P}}\right\}_{\mathrm{P}}-\left[Q_{0 M}^{P^{\prime}} Q_{0 P^{\prime}}^{N},\left[Q_{0 L}^{K}, Q_{0 P}^{P^{\prime \prime}} Q_{0 P^{\prime \prime}}^{Q}\right\}_{\mathrm{P}}\right\}_{\mathrm{P}}
\end{aligned}
$$

This is a super extension of the standard Serre relation in the bosonic case. It should be noted that the Serre relation is not modified up to the replacement of the commutator, while the index factors appear in the case of super extension of Jacobi identity. This fact implies that the structure of Hopf algebra is preserved under the super extension. As a final remark, the above Serre relation is not modified even if we replace $Q_{i M}^{N}$ by $\widehat{Q}_{i M}{ }^{N}$.

\subsection{Lax pair and transfer matrix}

Here, we shall consider the integrable structure of AdS-superstring.

\footnotetext{
${ }^{6}$ The global Symmetry of the AdS-superstring is PSL(4|4) as discussed by Metsaev and Tseytlin [43], and in the Roiban-Siegel formalism, auxiliary degrees of freedom for two GL(1)'s are introduced for the simpler linear realization of GL(4|4). The GL(1) symmetry is anomalous for the AdS-superstring and the D-branes classically by the WZ term [32]. The superparticle system, which governs the massless sectors of the $\mathcal{N}=4$ Yang-Mills system, contains manifest GL(4|4) symmetry including GL(1)'s in the RoibanSiegel formalism. The GL(1) may be broken in the quantum level, since the measure is not invariant under it. We are grateful to Nathan Berkovits and Warren Siegel for fruitful discussions on this point.
} 
An alternative to the iterative definition of the non-local charges is to utilize the Lax pair given by

$$
\begin{aligned}
& \left(L_{\tau}\right)_{M}^{N}(\sigma ; \lambda) \equiv \frac{\lambda}{\lambda^{2}-1}\left[\lambda\left(J_{\tau}^{R}\right)_{M}^{N}+\left(J_{\sigma}^{R}\right)_{M}^{N}\right] \\
& \left(L_{\sigma}\right)_{M}^{N}(\sigma ; \lambda) \equiv \frac{\lambda}{\lambda^{2}-1}\left[\lambda\left(J_{\sigma}^{R}\right)_{M}^{N}+\left(J_{\tau}^{R}\right)_{M}^{N}\right]
\end{aligned}
$$

The zero-curvature condition and the conservation law are equivalent to the condition:

$$
\left[\partial_{\tau}+L_{\tau}, \partial_{\sigma}+L_{\sigma}\right]=0
$$

By using the Lax pair, a transfer matrix $T\left(\sigma, \sigma^{\prime} ; \lambda\right)$ can be defined as a solution of the equation

$$
\left[\partial_{\sigma}+L_{\sigma}(\sigma ; \lambda)\right] T\left(\sigma, \sigma^{\prime} ; \lambda\right)=0, \quad T(\sigma, \sigma ; \lambda)=\mathbf{1} .
$$

The solution of $(3.11)$ is

$$
T\left(\sigma, \sigma^{\prime} ; \lambda\right)=\mathrm{P} \exp \left(-\int_{\sigma^{\prime}}^{\sigma} d \xi L_{\sigma}(\xi ; \lambda)\right)
$$

where the symbol $\mathrm{P}$ denotes the equal-time path ordering in terms of $\epsilon(\sigma)$. We can re-derive non-local charges by expanding the following relation:

$$
T(\lambda) \equiv T(2 \pi, 0 ; \lambda)=\exp \left(\sum_{n=0}^{\infty} \lambda^{n+1} Q_{n}\right) .
$$

The $Q_{0}$ and $Q_{1}$ are obtained from the first and second order parts with respect to $\lambda$, respectively. The gauge invariance of all non-local charges follows the fact that $T(2 \pi, 0 ; \lambda)$ commutes with the gauge generators.

It is possible to derive a classical $r$-matrix of the AdS-superstring by calculating the Poisson bracket of transfer matrices. Though we are confronted with the subtlety as in the case of principle chiral models ${ }^{7}$ [20] because of the non-ultra local terms, we can avoid it by following the prescription given in $[26,41,24]$. Indeed, after small algebra, we can readily show the following

\footnotetext{
${ }^{7}$ If there is no non-ultra local term, for example, in the case of generalized GrossNeveu model, then we do not encounter this subtlety [18]. The classical $r$-matrices are well-defined [18] and the classical Yang-Baxter equation is also satisfied [19].
} 
Poisson bracket for the $L_{\sigma}(\sigma ; \lambda)$ :

$$
\begin{aligned}
& {\left[\int\left(L_{\sigma}\right)_{M}^{N}(\sigma ; \lambda),\left(L_{\sigma}\right)_{L}^{K}\left(\sigma^{\prime} ; \mu\right)\right\}_{\mathrm{P}}} \\
& \quad=\frac{\lambda \mu}{\lambda-\mu}(-)^{N} \\
& \quad \times\left[\frac{1}{\mu^{2}-1}\left\{(-)^{(N+L)(1+M+L)} \delta_{M}^{K}\left(L_{\sigma}\right)_{L}^{N}\left(\sigma^{\prime} ; \lambda\right)-\delta_{L}^{N}\left(L_{\sigma}\right)_{M}^{K}\left(\sigma^{\prime} ; \lambda\right)\right\}\right. \\
& \left.\quad-\frac{1}{\lambda^{2}-1}\left\{(-)^{(N+L)(1+M+L)} \delta_{M}^{K}\left(L_{\sigma}\right)_{L}^{N}\left(\sigma^{\prime} ; \mu\right)-\delta_{L}^{N}\left(L_{\sigma}\right)_{M}^{K}\left(\sigma^{\prime} ; \mu\right)\right\}\right] .
\end{aligned}
$$

Following the paper [24], we can read off the $r$-matrix defined by

$$
r(\lambda, \mu)_{M L}^{N K} \equiv \frac{\lambda \mu}{\lambda-\mu} \delta_{L}^{N} \delta_{M}^{K}(-)^{N K} .
$$

This $r$-matrix is well-defined and obeys the classical Yang-Baxter equation,

$$
r(\lambda, \mu)_{M Q}^{N P} r(\mu, \nu)_{Q L}^{P K}+r(\mu, \nu)_{M Q}^{N P} r(\nu, \lambda)_{Q L}^{P K}+r(\nu, \lambda)_{M Q}^{N P} r(\lambda, \mu)_{Q L}^{P K}=0,
$$

as it is ensured by the structure of $Y(4 \mid 4)$. Thus the classical integrability of the AdS-superstring has been shown.

\section{Conclusion and discussion}

We have discussed a classical integrability of the type IIB superstring theory on the $\mathrm{AdS}_{5} \times \mathrm{S}^{5}$ background from the viewpoint of Yangian symmetry. The Roiban-Siegel formulation has been used to explicitly construct an infinite set of non-local charges, the first two of which are the generators of the Yangian algebra. We have constructed explicitly non-local charges by carefully treating the Wess-Zumino term and the constraint conditions intrinsic to the AdS-superstring. The obtained non-local charges are $\kappa$-invariant as well as the gauge invariant. The Hamiltonian (2.15) also commutes with these non-local charges.

The existence of the fermionic constraints requires the local gauge group to be supergroup as derived in (2.23) and (2.25). Since the direct origin of the fermionic components of gauge connection $\mathcal{A}$ is the fermionic constraints, this local supergroup may be related to the local constraints algebra which is shown to exist in the AdS-superstring [31] as well as in the flat superstring. However, this local gauge group is the stability group of the coset at least for the bosonic part, so this supergroup is a result of the fact that the supercoset space is not a symmetric space. The WZ term effectively makes the stability group to be super-stability group which was discussed in [34]. 
Then we have calculated the super Yangian algebra and the Serre relation has been also shown. It remains to clarify whether the finite size effect of the string world-sheet surely breaks the Yangian symmetry or not. The method of [49] would be favourable in this line. In addition, we have constructed the transfer matrix from the Lax pair. From the Poisson bracket of transfer matrices, we can read off the classical $r$-matrix satisfying the classical YangBaxter equation. The classical integrability of the AdS-superstring may survive and imply the quantum integrability. It is also expected that the lattice quantization of our super Yangian would be connected to the super Yangian symmetry in $\mathcal{N}=4 \mathrm{SYM}_{4}$, through the procedure of [38]. We hope to report in detail on the classical and quantum integrabilities in our formulation in the future.

It is an interesting problem to consider the semi-classical quantization of AdS-superstring $[27,29]$ in the Roiban-Siegel formulation, by considering several classical solutions. In fact, we can embed several bosonic classical solution rewritten in the matrix variables [5] into this formulation. When we construct the classical solutions in the full analysis, it is expected to obtain the super classical spin chain corresponding to the super spin chain in the SYM side [7]. It is also interesting to study the Penrose limit [46] of the super Yangian algebra. Penrose limit of the Yangian algebra is discussed in [2]. The resulting algebra after taking the Penrose limit is expected to be obtained via the Inönü-Wigner contraction of our super Yangian algebra, as in the case of superalgebra between AdS and pp-wave [33]. The pp-wave limit of the charges in the Roiban-Siegel formulation was demonstrated in the $\mathrm{AdS}_{2} \times \mathrm{S}^{2}$ background in [30]. We will report these issues in the near future as another publication.

Obviously, it is quite important to consider the quantum super Yangian algebra by quantizing the non-local charges by following the original work of Lüscher and Pohlmeyer [37]. If the classical Yangian symmetry survives under the quantization, we can expect that the quantum AdS-superstring theory is drastically restricted by the quantum Yangian and may be exactly solvable. The matching of the spectra and the equivalence of the integrable structures between the spin chain and the string theories are confirmed up to the two-loop order [3] on a specific example. In fact, the Bethe equations for the diagonalization of the Hamiltonian of quantum strings on $\mathrm{AdS}_{5} \times \mathrm{S}^{5}$ at large string tension are proposed in [4]. It is also interesting in the direction for studies of the quantum AdS-superstring to investigate the beta-function and conformal fixed point by following the scenario recently presented by Polyakov [47]. The beta-function of non-linear sigma model with the super Lie group as the target space, for example, in the case of PSL(4|4), is also discussed by Bershadsky et al. [14]. In the case of PSL(4|4), the beta-function 
is zero due to the vanishing dual Coxeter number. In preparing our work, the relationship between the integrability of AdS-string and the integrable models was also discussed in [35].

We believe that the classical and quantum integrabilities will play an important role in studies of the AdS/CFT correspondence at non-BPS sectors.

\section{Acknowledgments}

We would like to thank H. Fuji, K. Kamimura, M. Sakaguchi, Y. Satoh, Y. Susaki, Y. Takayama, D. Tomino, and A. Yamaguchi for useful discussion. We would like to thank N. Berkovits and W. Siegel for fruitful discussions, especially on the $\kappa$-symmetry and the GL(1) symmetry. We appreciate the referee for fruitful and helpful suggestions for improving the manuscript. The work of K.Y. is supported in part by JSPS Research Fellowships for Young Scientists.

\section{Appendix A. GL $(n \mid n), \operatorname{SL}(n \mid n)$, and $\operatorname{PSL}(n \mid n)$}

In this place, we shall briefly introduce several properties of the super Lie group, $\operatorname{GL}(n \mid n), \operatorname{SL}(n \mid n)$ and $\operatorname{PSL}(n \mid n)$. The knowledge of these super groups helps us to understand the motivation of the Roiban-Siegel formulation and it is also applicable to other consideration in the AdS/CFT correspondence with the supermatrix formulation (for example, see $[14,11]$ ).

The supergroups $\operatorname{GL}(n \mid n)$ and $\operatorname{SL}(n \mid n)$ are defined as, respectively,

$$
\begin{aligned}
\operatorname{GL}(n \mid n) & :=\{M \in \operatorname{Mat}(n \mid n) \mid \operatorname{sdet} M \neq 0\}, \\
\mathrm{SL}(n \mid n) & :=\{M \in \operatorname{Mat}(n \mid n) \mid \operatorname{sdet} M=1\} .
\end{aligned}
$$

Here the $M \in \operatorname{Mat}(n \mid n)$ describes a $2 n \times 2 n$ supermatrix:

$$
M=\left(\begin{array}{ll}
A & B \\
C & D
\end{array}\right)
$$

where $A$ and $D$ in the diagonal parts are bosonic matrices and $B$ and $C$ in the off-diagonal parts are fermionic ones. The super Lie algebra of $\mathrm{SL}(n \mid n)$ is

$$
\operatorname{sl}(n \mid n):=\{M \in \operatorname{Mat}(n \mid n) \mid \operatorname{STr} M=0\},
$$

where the supertrace of $M$ is defined as

$$
\mathrm{S} \operatorname{Tr} M=\operatorname{Tr} A-\operatorname{Tr} D .
$$


The $\mathrm{SL}(n \mid n)$ has a $\mathrm{U}(1)$ subgroup as a non-trivial centre, and so it is not simple. A generator proportional to the identity matrix $\alpha \cdot \mathbf{1}_{2 n}\left(\alpha \in \mathbb{C}, 1_{2 n} \in\right.$ $\operatorname{sl}(n \mid n))$ is included in the $\operatorname{sl}(n \mid n)$. This generator gives the $\mathrm{U}(1)$ symmetry. Then we can define the super Lie group $\operatorname{PSL}(n \mid n) \equiv \mathrm{SL}(n \mid n) / \mathrm{U}(1)$ and it is simple. The $\operatorname{PSL}(n \mid n)$, however, does not have a representation in $\operatorname{Mat}(n \mid n)$. Notably, the $\operatorname{psl}(n \mid n)$ cannot be embedded into $\operatorname{sl}(n \mid n)$ as a subalgebra. As a comment, the above-mentioned centre does not exist in the case of $\operatorname{sl}(m \mid n)(m \neq n)$ that is simple.

The super Lie algebra $\operatorname{gl}(n \mid n)$ for the supergroup $\operatorname{GL}(n \mid n)$ has a nondegenerate metric given by $g_{i j} \equiv \mathrm{S} \operatorname{Tr}\left(T_{i} T_{j}\right)$, where $T_{i}$ are the generators of $\operatorname{gl}(n \mid n)$ in the fundamental representation. There are $(2 n)^{2}$ generators of $\operatorname{gl}(n \mid n)$, and we can choose as follows:

- supertraceless and traceless matrices $T_{a}\left(a=1, \ldots,(2 n)^{2}-2\right)$

- the identity $I \equiv 1_{2 n}$ and the matrix $J=\operatorname{diag}(1, \ldots, 1,-1, \ldots,-1)$.

By combining the $T_{a}$ with the identity $I$, the $\operatorname{sl}(n \mid n)$ is generated. In this basis, the $\mathrm{gl}(n \mid n)$ metric is given by

$$
g_{i j}=\left(\begin{array}{ccc}
g_{a b} & 0 \\
0 & 0 & 2 n \\
& 2 n & 0
\end{array}\right),
$$

where $g_{a b}$ is an invariant metric on $\operatorname{psl}(n \mid n)$.

\section{Appendix B. Treatment of non-ultra local terms}

The Poisson bracket of the AdS-string in the Roiban-Siegel formulation contains a subtlety for the non-ultra local term (i.e., the delta function term with a derivative) as in the principle chiral model.

The presence of the non-ultra local term in Poisson brackets of the currents may lead to the ambiguity of the Poisson brackets of non-local charges. Hence, we need to determine the regularization of the charges and the order of taking the limits, in order to obtain the well-defined Poisson brackets [37]. The subtlety of the limits in the principle chiral model is also discussed in [37, 39].

Here it is convenient in the parameterization of world-sheet, $-\pi \leq \sigma \leq$ $+\pi$, since the treatment of non-ultra local term can be discussed in the same way as in principle chiral models on a flat plane. We do not loose the generality even if we consider in this parameterization, because this 
parameterization can be mapped to other one via the translation of $\sigma$. For example, the shift of $\sigma, \sigma \rightarrow \sigma+\pi$ leads to the range $0 \leq \sigma \leq 2 \pi$.

In general, one can regularize the integral of the charges as

$$
\begin{aligned}
& Q_{0 m}^{n}= \lim _{X_{1}, X_{2} \rightarrow \pi} \int_{-X_{2}}^{X_{1}} d \sigma\left(J_{\tau}^{R}\right)_{m}^{n}(\sigma), \\
& Q_{1 m}^{n}=\lim _{Y_{1}, Y_{2} \rightarrow \pi}\left[\int_{-Y_{2}}^{Y_{1}} d \sigma J_{\sigma m}^{R}{ }^{n}(\sigma)\right. \\
&\left.\quad-\int_{-Y_{2}}^{Y_{1}} d \sigma \int_{-Y_{2}}^{Y_{1}} d \sigma^{\prime} \epsilon\left(\sigma-\sigma^{\prime}\right)\left[\left(J_{\tau}^{R}\right)(\sigma),\left(J_{\tau}^{R}\right)\left(\sigma^{\prime}\right)\right]_{m}^{n}\right],
\end{aligned}
$$

but then we have the ambiguity proportional to

$$
\lim _{X_{1}, X_{2} \rightarrow \pi} \lim _{Y_{1}, Y_{2} \rightarrow \pi}\left[\theta\left(Y_{1}-X_{1}\right)-\theta\left(Y_{2}-X_{2}\right)\right]
$$

in the Poisson bracket of $Q_{0}$ and $Q_{1}$. We can avoid this ambiguity by taking the regularization as $X_{1}=X_{2} \equiv X$ and $X_{1}=Y_{2} \equiv Y$. In other words, we can well define the Poisson bracket of $Q_{0}$ and $Q_{1}$ by taking this regularization.

In addition, there is an ambiguity with respect to the order of the limits exists in calculating the $Q_{0}$ term in the expression of $Q_{2},(3.7)$, and we may take three kinds of limits: (1) $\lim _{Y \rightarrow \pi}$ before $\lim _{Y^{\prime} \rightarrow \pi}$, (2) $\lim _{Y^{\prime} \rightarrow \pi}$ before $\lim _{Y \rightarrow \pi}$, and (3) $Y=Y^{\prime}$, where $Y$ and $Y^{\prime}$ are cutoffs for the left and right $Q_{1}$ in the Poisson bracket at the l. h. s. of (3.6). It is not, however, troublesome because all prescriptions give the same result $-4 Q_{0}$, i.e., the factor -4 is universal.

Finally, let us consider the subtlety that appears in the calculation of the Serre relation. In that time, we need to take the limit $\lim _{X \rightarrow \pi}$ before $\lim _{Y \rightarrow \pi}$. If we take limits inversely, then the additional terms, proportional to $Q_{0}$,

$$
-4 \delta_{l}^{q}\left(\delta_{m}^{k} Q_{0 p}^{n}-\delta_{p}^{n} Q_{0 m}^{k}\right)+4 \delta_{p}^{k}\left(\delta_{m}^{q} Q_{0 l}^{n}-\delta_{l}^{n} Q_{0 m}^{q}\right),
$$

appear and so the Serre relation is not satisfied. Thus, it is necessary to determine the order of the limits as above for the well-defined Yangian algebra. In other words, the Serre relation (namely the closure of the Yangian algebra) leads to the definite calculus of the Poisson bracket without imposing some artificial prescription by hand. The relationship of the Serre relation and the definition of Poisson bracket are discussed by MacKay [39] in the case of a principle chiral model.

As a matter of course, the regularization prescription is not modified when we consider the supermatrix case. 


\section{References}

[1] E. Abdalla, M.C.B. Abdalla and K. Rothe, Non-perturbative methods in two-dimensional quantum field theory, Singapore, World Scientific, 1991.

[2] L.F. Alday, Nonlocal charges on $A d S_{5} \times \mathrm{S}^{5}$ and pp-waves, JHEP 0312 (2003) 033, hep-th/0310146.

[3] G. Aruryunov and M. Staudacher, Matching higher conserved charges for strings and spins, JHEP 0403 (2004) 004, hep-th/0310182;

G. Aruryunov and M. Staudacher, Two-loop commuting charges and the string / gauge duality, hep-th/0403077.

[4] G. Arutyunov, S. Frolov and M. Staudacher, Bethe ansatz for quantum strings, JHEP 0410 (2004) 016, hep-th/0406256.

[5] G. Arutyunov, S. Frolov, J. Russo and A.A. Tseytlin, Spinning strings in $A d S_{5} \times \mathrm{S}^{5}$ and integrable systems, Nucl. Phys. B 671 (2003) 3, hepth/0307191;

G. Arutyunov, J. Russo and A.A. Tseytlin, Spinning strings in $A d S_{5} \times$ $\mathrm{S}^{5}$ : New integrable system relations, Phys. Rev. D 69 (2004) 086009, hep-th/0311004.

[6] N. Beisert, The complete one-loop dilatation operator of $N=4$ super Yang-Mills theory, Nucl. Phys. B 676 (2004) 3, hep-th/0307015.

[7] D. Beisert and M. Staudacher, The $\mathcal{N}=4 S Y M$ integrable super spin chain, Nucl. Phys. B670 (2003) 439, hep-th/0307042.

[8] N. Beisert, S. Frolov, M. Staudacher and A.A. Tseytlin, Precision spectroscopy of AdS/CFT, JHEP 0310 (2003) 037, hep-th/0308117.

[9] I. Bena, J. Polchinski and R. Roiban, Hidden symmetries of the $A d S_{5} \times$ $\mathrm{S}^{5}$ superstring, Phys. Rev. D 69 (2004) 046002, hep-th/0305116.

[10] D. Berenstein, J.M. Maldacena and H. Nastase, Strings in flat space and pp waves from $N=4$ super Yang Mills, JHEP 0204 (2002) 013, hep-th/0202021.

[11] N. Berkovits, M. Bershadsky, T. Hauer, S. Zhukov and B. Zwiebach, Superstring theory on $A d S_{2} \times \mathrm{S}^{2}$ as a coset supermanifold, Nucl. Phys. B 567 (2000) 61, hep-th/9907200.

[12] D. Bernard, Hidden Yangians in 2-D massive current algebras, Commun. Math. Phys. 137 (1991) 191.

[13] D. Bernard, An introduction to Yangian symmetries, Int. J. Mod. Phys. B 7 (1993) 3517, hep-th/9211133.

[14] M. Bershadsky, S. Zhukov and A. Vaintrob, $P S L(n \mid n)$ sigma model as a conformal field theory, Nucl. Phys. B 559 (1999) 205, hep-th/9902180. 
[15] E. Brezin, C. Itzykson, J. Zinn-Justin and J.B. Zuber, Remarks about the existence of nonlocal charges in two-dimensional models, Phys. Lett. B 82 (1979) 442.

[16] C. Briot and E. Ragoucy, W-superalgebras as truncation of superYangians, math.QA/0209339.

[17] T.L. Curtright and C.K. Zachos, Nonlocal currents for supersymmetric nonlinear models, Phys. Rev. D 21 (1980) 411; T.L. Curtright and C.K. Zachos, Supersymmetry and the nonlocal yangian deformation symmetry, Nucl. Phys. B 402 (1993) 604, hep-th/9210060;

L.E. Saltini and A. Zadra, Algebra of non-local charges in supersymmetric non-linear sigma models, Int. J. Mod. Phys. A 12 (1997) 419, solv-int/9511007.

[18] H.J. de Vega, H. Eichenherr and J.M. Maillet, Canonical charge algebras for integrable fermionic theories, Phys. Lett. B 132 (1983) 337.

[19] H.J. de Vega, H. Eichenherr and J.M. Maillet, Yang-Baxter algebras of monodromy matrices in integrable quantum field theories, Nucl. Phys. B 240 (1984) 377.

[20] H.J. de Vega, H. Eichenherr and J.M. Maillet, Classical and quantum algebras of nonlocal charges in sigma models, Commun. Math. Phys. 92 (1984) 507.

[21] L. Dolan, Kac-Moody algebra is hidden symmetry of chiral models, Phys. Rev. Lett. 47 (1981) 1371;

J.H. Schwarz, Classical symmetries of some two-dimensional models, Nucl. Phys. B 447 (1995) 137, hep-th/9503078.

[22] L. Dolan, C.R. Nappi and E. Witten, A relation between approaches to integrability in superconformal Yang-Mills theory, JHEP 0310 (2003) 017, hep-th/0308089; L. Dolan, C.R. Nappi and E. Witten, Yangian symmetry in $D=4$ superconformal Yang-Mills theory, Contributed to 3rd International Symposium on Quantum Theory and Symmetries (QTS3), Cincinnati, Ohio, 10-14 Sep 2003, hep-th/0401243.

[23] V. Drinfel'd, Hopf algebras and the quantum Yang-Baxter equation, Dokl. Acad. Nauk. USSR, 283 (1985) 1060;

V. Drinfel'd, Hopf algebras and the quantum Yang-Baxter equation, Sov. Math. Dokl. 32 (1985) 254;

V. Drinfel'd, A new realization of yangians and quantized affine algebras, Sov. Math. Dokl. 36 (1988) 212.

[24] A. Duncan, H. Nicolai and M. Niedermaier, On the poisson bracket algebra of monodromy matrices, Z. Phys. C 46 (1990) 147.

[25] J.M. Evans, N.J. MacKay and M. Hassan, Conserved charges and supersymmetry in principal chiral models, hep-th/9711140; 
J.M. Evans, M. Hassan, N.J. MacKay and A.J. Mountain, Local conserved charges in principal chiral models, Nucl. Phys. B 561 (1999) 385, hep-th/9902008;

J.M. Evans, N.J. MacKay and M. Hassan, Conserved charges and supersymmetry in principal chiral and WZW models, Nucl. Phys. B 580 (2000) 605, hep-th/0001222.

[26] L.D. Faddeev and N.Y. Reshetikhin, Integrability of the principal chiral field model in $(1+1)$-dimension, Annals Phys. 167 (1986) 227.

[27] S. Frolov and A.A. Tseytlin, Semiclassical quantization of rotating superstring in $A d S_{5} \times S^{5}$, JHEP 0206 (2002) 007, hep-th/0204226.

[28] S.S. Gubser, I.R. Klebanov and A.M. Polyakov, Gauge theory correlators from non-critical string theory, Phys. Lett. B 428 (1998) 105, hep-th/9802109.

[29] S.S. Gubser, I.R. Klebanov and A.M. Polyakov, A semi-classical limit of the gauge/string correspondence, Nucl. Phys. B 636 (2002) 99, hepth/0204051.

[30] M. Hatsuda, AdS and pp-wave D-particle superalgebras, JHEP 0312 (2003) 016, hep-th/0302027.

[31] M. Hatsuda and K. Kamimura, Classical AdS superstring mechanics, Nucl. Phys. B 611 (2001) 77, hep-th/0106202.

[32] M. Hatsuda and K. Kamimura, Wess-Zumino terms for AdS D-branes, Nucl. Phys. B 703 (2004) 277, hep-th/0405202.

[33] M. Hatsuda, K. Kamimura and M. Sakaguchi, Super-PP-wave algebra from super-AdS $\times S$ algebras in eleven-dimensions, Nucl. Phys. B 637 (2002) 168, hep-th/0204002; M. Hatsuda, K. Kamimura and M. Sakaguchi, From super-AdS $S_{5} \times \mathrm{S}^{5}$ algebra to super-pp-wave algebra, Nucl. Phys. B 632 (2002) 114, hep-th/0202190.

[34] M. Hatsuda and W. Siegel, A new holographic limit of $A d S_{5} \times \mathrm{S}^{5}$, Phys. Rev. D67 (2003) 066005, hep-th/0211184.

[35] B.Y. Hou, D.T. Peng, C.H. Xiong and R.H. Yue, The affine hidden symmetry and integrability of type IIB superstring in $A d S_{5} \times \mathrm{S}^{5}$, hepth/0406239,

B.Y. Hou, B.Y. Hou, X.H. Wang, C.H. Xiong and R.H. Yue, Conformal affine Toda soliton and moduli of IIB superstring on $A d S_{5} \times \mathrm{S}^{5}$, hepth/0406250; B.Y. Hou, D.T. Peng, C.H. Xiong and R.H. Yue, The affine ambitwistor space as the moduli space of $S U Y M$ in $A d S_{5} \times \mathrm{S}^{5}$, hep-th/0406251.

[36] V.E. Korepin, N.M. Bogoliubov and A.G. Izergin, Quantum inverse scattering method and correlation functions Cambridge University Press, Cambridge, United Kingdom, 1993; 
For the periodic boundary conditions in a quantum spin chain, see F.D.M. Haldane, Z.N.C. Ha, J.C. Talstra, D. Bernard and V. Pasquier, Yangian symmetry of integrable quantum chains with long range interactions and a new description of states in conformal field theory, Phys. Rev. Lett. 69 (1992) 2021.

[37] M. Lüscher, Quantum nonlocal charges and absence of particle production in the two-dimensional nonlinear sigma model, Nucl. Phys. B 135 (1978) 1;

M. Lüscher and K. Pohlmeyer, Scattering of massless lumps and nonlocal charges in the two-dimensional classical nonlinear sigma model, Nucl. Phys. B 137 (1978) 46.

[38] N.J. MacKay, Lattice quantization of Yangian charges, Phys. Lett. B 349 (1995) 94, hep-th/9501079.

[39] N.J. MacKay, On the classical origins of Yangian symmetry in integrable field theory, Phys. Lett. B 281 (1992) 90; N.J. MacKay, On the classical origins of Yangian symmetry in integrable field theory, Phys. Lett. B 308 (1993) 444.

[40] G. Mandal, N.V. Suryanarayana and S.R. Wadia, Aspects of semiclassical strings in $A d S_{5}$, Phys. Lett. B 543 (2002) 81, hep-th/0206103.

[41] J.M. Maillet, New integrable canonical structures in two-dimensional models, Nucl. Phys. B 269 (1986) 54.

[42] J.M. Maldacena, The large $N$ limit of superconformal field theories and supergravity, Adv. Theor. Math. Phys. 2 (1998) 231; J.M. Maldacena, The large $N$ limit of superconformal field theories and supergravity, [Int. J. Theor. Phys.] 38 (1999) 1113, hep-th/9711200.

[43] R.R. Metsaev and A.A. Tseytlin, Type IIB superstring action in $A d S_{5} \times$ $S_{5}$ background, Nucl. Phys. B 533 (1998) 109, hep-th/9805028.

[44] J.A. Minahan and K. Zarembo, The Bethe-ansatz for $N=4$ super Yang-Mills, JHEP 0303 (2003) 013, hep-th/0212208.

[45] M.L. Nazarov, Quantum Berezinian and the classical Capelli identity, Lett. Math. Phys. 21 (1991) 123.

[46] R. Penrose, Any spacetime has a plane wave as a limit, Differential geometry and relativity, Reidel, Dordrecht, 1976, pp. 271-275.

R. Gueven, Plane wave limits and T-duality, Phys. Lett. B 482 (2000) 255, hep-th/0005061.

[47] A.M. Polyakov, Conformal fixed points of unidentified gauge theories, Mod. Phys. Lett. A19 (2004) 1649, hep-th/0405106.

[48] R. Roiban and W. Siegel, Superstrings on $A d S_{5} \times S^{5}$ supertwistor space, JHEP 0011 (2000) 024, hep-th/0010104. 
[49] K. Schoutens, Yangian symmetry in conformal field theory, Phys. Lett. B 331 (1994) 335, hep-th/9401154.

[50] J.S. Schwinger, Field theory commutators, Phys. Rev. Lett. 3 (1959) 296.

[51] E. Witten, Anti-de Sitter space and holography, Adv. Theor. Math. Phys. 2 (1998) 253, hep-th/9802150.

[52] R.B. Zhang, The $g l(M \mid N)$ super Yangian and its finite dimensional representations, Lett. Math. Phys. 37 (1996) 419, q-alg/9507029. 